\section{Reducing fetal radiation dose in computed tomography for pregnant patients: $A$ literature review}

\section{Hamid Ghaznavi}

Department of Radiology, Faculty of Paramedical Sciences, Kurdistan University of Medical Sciences, Sanandaj, Iran.
Address for correspondence:

Hamid Ghaznavi, Department of Radiology, Faculty of Paramedical Sciences, Kurdistan University of Medical Sciences, Sanandaj, Iran. Email: hamid.ghaznavi@muk.ac.ir

How to cite this article: Ghaznavi H. Reducing fetal radiation dose in computed tomography for pregnant patients: A literature review.

G Med Sci. 2021; 2(4): 035-043.

https://www.doi.org/10.46766/thegms.radiology.21082006

Copyright: @ 2021 Hamid Ghaznavi. This is an Open Access article distributed under the Creative Commons Attribution License, which permits unrestricted use, distribution, and reproduction in any medium, provided the original work is properly cited.

\title{
Abstract
}

To diagnose diseases during gestation period including renal stones, appendicitis, and pulmonary embolism in pregnant patients, computed tomography (CT) can be a golden standard. Due to CT examination, the fetus is prone to receiving a considerable dose which is the result of direct or scattered (external and internal scattered radiation) beams. The effects of ionization radiation on fetus include mutagenesis and carcinogenesis, therefore, it is essential to reduce fetus dose for pregnant patients who undergo CT examination during gestation period. This article aims to review approaches that are effective in reducing fetal dose in pregnant patients.

\section{Introduction}

Performing computed tomography (CT) in gestation period has increased considerably by $25 \%$ per year, Pulmonary embolism, renal colic, ovarian torsion, and appendicitis are common indications for CT in pregnant patients [1]. The effects of ionization radiation on fetus include Mutagenesis, teratogenesis, and carcinogenesis. It has been demonstrated that the dose threshold for fetal effects is below 100-200 mGy, and fetal dose below this amount is not associated with measurable increase in non-cancer fetal risks and teratogenesis [2].

The safest diagnostic imaging procedure in pregnancy is ultrasound, but it occasionally fails, or maybe it is not available, in that case, СТ becomes the modality of choice. The reasons CT is the best choice in this situation are that it is fast, reliable, and less susceptible to equivocal error [3]. Fetal absorbed doses resulting from chest CT, brain $\mathrm{CT}$, pulmonary $\mathrm{CT}$, abdomen $\mathrm{CT}$, and abdomen pelvis $\mathrm{CT}$ are $0.2,00.1,0.2,4$, and $25 \mathrm{mGy}$, respectively, whereas background dose during 9 months of pregnancy is $0.5-1$ mGy [4]. Generally, severe strategies have been introduced for reducing doses in CT examinations. These strategies include using tube current modulation and automatic exposure control to adapt mAs to patient size, using appropriate $\mathrm{kV}$ based on clinical indication and patient size, using non-overlapping pitch to minimize motion artifacts, limiting scan volume, using lead shielding, and iterative reconstruction [5]. with the growing fetus size and its movement closer to scan volume, the fetal dose increases, therefore, fetus dose changes during gestation period in CT examinations [6].

American College of Radiology (ACR) proposed guidelines for pregnant patients undergoing CT examinations. It is recommended that necessary CT examinations be performed just after clinical work-up, and radiation exposure be kept as low as reasonably achievable (known as ALARA principles). In addition, counseling is essential for performing CT in pregnant patients. It should be noted that iodinated contrast agents are safe in pregnancy [7]. If performing contrast agents is necessary, high-speed injection should be performed because of high cardiac output of pregnant patients [8].

Appropriate techniques can reduce fetal dose of pregnant patients who undergo CT. These techniques include 
changing the scan parameter and scan volume, avoiding multiple phases examination, performing shielding, using sophisticated reconstruction algorithms, and avoiding direct exposure of the fetus especially in scout image and elimination of lateralscoutimage $[9,10]$. Figure1illustrated the algorithms for performing diagnostic procedures in pregnant patients with pulmonary embolism (PE) [9], appendicitis, and urolithiasis [10]. Sometimes because of life threatening conditions, performing CT for pregnant patients is necessary. In this situation, minimizing the radiation dose of fetus is very important. The aim of this study is to review studies of using approaches for reducing fetal dose and its effectiveness in CT examination for pregnant patients.

\section{Methods and Materials}

During April 2021 PubMed and Medline were searched for "fetus [MESH]" and "pregnancy [MESH]" and "computed tomography [MESH]" and "pregnant women [MESH]" and "radiation dosage [MESH]", to retrieve papers published on fetal dose in computed tomography for pregnant patients. Articles in English language were reviewed. References of the articles were screened for other papers and included in this review when considered relevant. The inclusion criteria in this paper includes published papers about reducing fetal dose in ionizing radiation procedures for pregnant patients. But the exclusion criteria conducted by eliminating papers which evaluated approaches for reducing fetus dose in conventional and interventional radiology, mammography, and fluoroscopy. In fact, only clinical investigations which evaluated approach to reduce fetal dose in CT scans were selected for this paper.

\section{Results}

\subsection{Main approaches to reduce dose in CT}

\section{A. Tube Current Modulation}

Tube Current Modulation (TCM) refers to the control of the $\mathrm{mA}$ in two directions of the patient (Longitudinal and Angular) during CT examination. TCM takes into consideration the patient volume and the attenuation differences of various tissues. Due to this technique, the patient dose is significantly reduced compared to manual selection of mA. TCM is the best approach to reduce patient dose in CT, the reason for this is the adjustment of the dose according to the patient's attenuation in angular and longitudinal directions [11]. Automatic exposure control (AEC) is an installed technology that adjusts the tube current automatically in response to variations in $\mathrm{x}$-ray intensity at the detector. It is found that AEC reduces radiation dose up to $40 \%$ [5]. Assessment of fetal doses after CT examinations using Monte Carlo method in three types of realistic computations at the end of 3,6 and 9 months of pregnancy showed that TCM reduced fetal doses by $14 \%, 18 \%$ and $25 \%$ respectively [12].

\section{B. Scan parameters}

Ibal et al., investigated the effects of scan parameter on fetal dose [13]. As $\mathrm{kVp}$ and $\mathrm{mAs}$ are raised, received dose increases directly. The percentage of dose reduction depends on beam energy, meaning that with the constant thickness of lead apron, dose reduction in high $\mathrm{kVp}$ is smaller than low $\mathrm{kVp}$. The effect of $\mathrm{kVp}$ on increasing patient dose is more considerable than $\mathrm{mAs}$ because fetal dose is proportional to the square of the $\mathrm{kVp}$. It should be noted that excessive reduction of $\mathrm{kVp}$ and $\mathrm{mAs}$ is not acceptable because it leads to an increase in the image noise. Based on diagnostic indication, rotation time can be varied, but it was found that it has no significant effect in changing the fetal dose. Minimizing the pitch can reduce the fetal dose slightly. The reason that how increasing pitch can reduce fetal dose is changing the distance between the edge of scanned volume and the fetus position. Furthermore, collimation has the same effect, and by increasing the collimation of the beam, the fetal dose will be increased.

\section{External shielding}

Lead shielding, known as external shielding, can be used to decrease the fetal dose during pulmonary CT scan. Routine protocol for using lead shielding for pregnant patients during pulmonary CT includes a lead apron placed above the pregnant patient and one under her; and the thickness of these aprons is $0.7 \mathrm{~mm}$. In addition to the above and under aprons, one lead apron with $0.35 \mathrm{~mm}$ thickness on either side is placed around the patient. These aprons are positioned from lower costal margin or bottom of the rib to cover the abdomen and pelvis. It was found that fetal dose will be reduced up to $47 \%$ by using a $0.7 \mathrm{~mm}$ lead apron [9].

Using abdominal lead shielding on pregnant patients undergoing CT scans has been investigated. The highest and the lowest use of lead shielding across the world were related to North America (94.5\%) and Europe (46.3\%), respectively. There are several reasons for not using lead shielding in case of pregnant patients. One of these reasons was the back pain of pregnant patients, and another reason was these patients' complaints about the weight of the lead shielding. These complaints are the consequences of inflexibility and lack of a functional design for shielding purposes in pregnant patients [14]. Figure 2 illustrates the impact of lead shielding on reducing fetal dose. 
Monte Carlo calculation has evaluated the use of external shielding for reducing fetal dose in chest and abdominal CT. Results revealed that using lead shielding with 0.7 mm thickness could reduce the fetal dose up to $56 \%$. In addition, for chest and upper abdominal CT, reducing the scan length by one to three centimeters could reduce fetal dose up to $24 \%$ and $47 \%$, respectively [15].

Bismuth shield is another external shielding which can be used for dose reduction in CT examination. Through attenuating primary $\mathrm{x}$-ray beams, this shield reduces dose to superficial radiosensitive organs in scan volumes such as eyes, thyroid and breast in brain, cervical, and chest CT, respectively, although it affects the image quality by increasing the noise under the shield and the absorption of photons that exit from the patients. Greater attenuation, especially at higher tube potential, and flexibility compared to lead shields are the major advantages of bismuth shields [16-19].

A comparison between lead and bismuth-antimony Shield for Reducing Fetal Dose shows that lead shields reduce dose more than bismuth-antimony shields (73\% vs $62 \%$ ) by using $120 \mathrm{kVp}$ in CT pulmonary angiography. Due to using $100 \mathrm{kVp}$, lead shields reduce dose more than the bismuth-antimony shields $(72 \%-79 \%$ vs $57 \%-81 \%)$. In fact, the effectiveness of reducing voltage and limiting scan volume is more than external shielding at reducing fetal dose, and there is no significant difference between lead and bismuth antimony shields in reducing fetal dose when scan parameters are optimized [20]. Figure 3 illustrates the diagram of sources of scattered radiation that contribute to the fetal dose [21].

\section{Internal shielding}

This shielding is a new approach for deceasing fetal dose in chest and pulmonary CT in which the pregnant patient slowly swallows 1-2 glasses of Barium Sulphate on CT table. Phantom study showed that internal shields are more effective in reducing fetal dose from chest CT, compared to external shielding. Calculations show that internal scatter in the near field was reduced $13 \%$ and $87 \%$ by administering $2 \%$ and $40 \%$ barium sulfate. Moreover, experimental measurements using TLD showed that administering $40 \%$ barium sulpate reduces internal scatter up to $98 \%$ in the near field. Internal scatter is approximately reduced $90 \%$ by performing barium sulphate. The percentage of dose reduction using barium sulphate is equal to $1 \mathrm{~mm}$ lead shield. To avoid artifact, it is recommended that patients drink water to wash out barium sulphate from esophagus after administering barium sulphate. This study seems to be innovative, but its results have been obtained from phantom study and have not been validated in human [22].

\section{E. Reconstruction Algorithm}

One of the important applications of reconstruction algorithm is decreasing radiation dose, but due to this advantage, image noise increases. Filtered back projection (FBP) was a common reconstruction algorithm in CT, but it was unable to reduce radiation dose while maintaining image quality. In fact, lowering radiation dose sparked an increase in image noise. Adaptive statistical iterative algorithm is a new approach in CT to reduce radiation dose while preserving image quality, compared to FBP, especially in low dose CT protocol [23, 24].

Assessment of low dose protocol using iterative reconstruction (IR) algorithm in abdominal CT examination showed that radiation dose was reduced up to $48 \%$ without affecting the image quality [25]. Comparison between deep learning image reconstruction (DLIR) algorithm, the latest introduced complex reconstruction algorithm, and hybrid iterative reconstruction (IR) algorithm to evaluate dose reduction while improving image quality revealed that potential of dose optimization in DLIR algorithm is more than hybrid IR algorithm, and image noise, detectability without changing the noise texture, and spatial resolution in acquired image by DLIR algorithm improved compared to hybrid IR algorithm [26]. DLIR algorithm has remained a fully operational technique to reduce radiation dose while maintaining image quality during CT examination in pregnant patients [27].

\subsection{Type of CT examinations in pregnancy and approaches to reduce fetal dose}

\section{A. Urinary diseases in pregnancy}

$10 \%$ of people may experience kidney stones during their lives, therefore, it can also happen for pregnant women during gestation period [28]. The incidence of kidney stone in gestation period is relatively rare, however, it was found that the incidence of this pathology is one per 204 pregnant women [29]. Ultrasound is frequently used as a diagnostic procedure for detecting renal pathologies, but CT is more sensitive than ultrasound for diagnosing stones. Using low tube current protocol, fetal dose for kidney stones CT ranging from 0 to 3 months of gestation is $4-7.2 \mathrm{mGy}$ to $8.5-11.7 \mathrm{mGy}$. The average fetal doses in the first trimester of gestation for abdominal and pelvic CT are calculated 23.57 mGy. Minimizing fetal dose in kidney stones CT requires limiting the scan volume, lowering the tube current (160 mAs) and increasing pitch [30, 31].

Low dose protocol was applied for abdominal and pelvic CT in 20 pregnant patients. The parameter in this protocol included $120 \mathrm{kVp}$, pitch:1.5, and mAs: 80-160 (just for two patients, mAs was 184 and 196). Evaluation of fetal dose in 
these patients showed that the mean calculated radiation dose was $7 \mathrm{mGy}$, and this protocol had considerable sensitivity for detecting stones in the urinary system [32].

A systematic review has investigated the accuracy of identification of urinary tract stones using low dose CT $(<3.5 \mathrm{mSv})$ and ultra-low dose CT $(<1.9 \mathrm{mSv})$. Radiation dose is particularly reduced by lowering $\mathrm{mA}$ and $\mathrm{kVp}$, and the impact of applying iterative reconstruction on reducing dose and increasing accuracy of identification stones was considerable. The diagnostic accuracies for low dose CT and ultra-low dose CT were $94.3 \%$ and $95.5 \%$, respectively. Therefore, it is recommend that these two protocols be performed for patients with urine tract stone instant of standard CT protocol [33].

Urolithiasis is another common non-obstetric disease in pregnant patients. Ultrasound remains the first-line imaging procedure for diagnosing this pathology. The specificity and sensitivity of ultrasound for detection urolithiasis are $86 \%$ and $34 \%$, respectively, but they over $98 \%$ with CT, therefore, CT is the golden standard for identifying the nephrolithiasis in pregnant patients [34]. Systematic evaluation of standard-dose, low-dose, and ultra-low dose CT of urinary system shows that the effective radiation dose using ultra-low dose CT ranges from 0.5 to $1.9 \mathrm{mSv}$ with sensitivity of $90-100 \%$ and specificity up to $100 \%$, therefore, images acquired by performing this protocol has a significant quality for detecting urolithiasis with much reduced radiation dose in pregnant patients $[35,36]$.

The guideline of urological association of Asia introduced CT as a last-line option after ultrasound and magnetic resonance imaging (MRI), and it is recommended that reducing radiation dose should be performed for patients with $\mathrm{BMI}<30 \mathrm{~kg}, \mathrm{~m}^{2}$ low dose CT (with dose $<4 \mathrm{mSv}$ ) [37].

To summarize, reducing fetal dose in urinary CT examination requires:
I. Lowering $\mathrm{mAs}(<160)$ and $\mathrm{kVp}(\leq 120)$
II. Limiting scan volume
III. Performing pitch $>1$
IV. Applying iterative reconstruction
V. Wider beam collimation (for increasing dose efficiency)

\section{B. Appendicitis in Pregnancy}

Appendicitis, which usually happens in third trimester of gestation, is another common disease during gestation. The incidence of appendicitis in pregnant patients increases by gestation age, and it happens 1 in 1500 pregnancies $[38,39]$. Evaluation of probability of accurate diagnosis of appendicitis using ultrasound and CT/ultrasound has revealed that ultrasound followed by CT significantly increases the accuracy of appendicitis diagnosis, therefore, unnecessary operations are reduced in these patients [40].

The approaches offered to reduce the fetal dose for the appendix CT are almost the same as those of the urinary CT (120 kV is general in appendix CT). Among them, limiting the scan volume is necessary, and it should be noted that dual-phase (with and without contrast agents) appendix CT examination is prohibited, except in very urgent circumstances [40].

Damilakis et al. measured fetal dose in abdominal CT by considering Automatic Exposure Control (AEC), and improper alignment of the pregnant patient at the gantry isocenter. Their results showed that activation of AEC where the phantom is placed on isocenter significantly reduces fetal dose at all gestational trimesters, but activating the AEC increases the image noise at all gestational trimesters, compared to fixed mAs. The effect of off-centering patient at the gantry isocenter is not considerable, but it can affect the image quality. Therefore, to preserve the image quality, it is recommended that pregnant patients be placed at the center of gantry [41].

To summarize, reducing fetal dose in appendix CT examination requires:

I. The same approach as that for urinary system CT

II. Single phase only

III. $120 \mathrm{kVp}$ generally

IV. Apply AEC

\section{Pulmonary embolism in pregnancy}

The incidence of pulmonary embolism in pregnant patients is 5-12 per 10,000 pregnancies [42]. An epidemiological study in a South Asian multi-ethnic country showed that the incidence of this disease was 18 cases per 174,708 pregnant patients [43]. The algorithm of imaging procedures for diagnosis of pulmonary embolism during pregnancy has been illustrated in figure 1. In pregnant patients with suspected pulmonary embolism, priority of using ionization radiation for diagnosing this pathology includes chest $\mathrm{x}$-ray, lung scintigraphy, and pulmonary embolism CT. Radiation doses during pulmonary embolism CT should be maintained under 50 mGy [9].

The main approach to reduce fetal dose in pulmonary embolism CT includes [44-47]:

I. Limiting scan volume

II. Patient centering is recommended to reduce fetal dose

III. Scout image should be performed at low $\mathrm{kVp}$ and low mAs for localizer radiograph, and lateral scout image should be eliminated

IV. Use $\mathrm{kVp} \leq 100$ (if patients $\mathrm{BMI}<30 \mathrm{~kg} / \mathrm{m}^{2}, \mathrm{kVp}$ can be reduced to $80 \mathrm{kVp}$ 
V. AEC technique is preferred, compared to fixed $\mathrm{mA}$

VI. Increase in pitch and detector collimation thickness

VII. Applying iterative reconstruction algorithm

VIII. Avoid scanning the pelvis and lower extremities for possible deep vein thrombosis (Venography CT)

IX. Use shielding (mostly scatter radiation which can be received by fetus is a result of internal scatter, therefore, to reduce this radiation, internal shielding can be more useful than external shielding. The reason for using lead shielding is just to decrease the patient's anxiety).

$\mathrm{X}$. Optimal administering the contrast agent in pulmonary arteries (bolus timing technique is recommended). The rate of injection should be increased $(5 \mathrm{ml} / \mathrm{s})$ because of high cardiac output during gestation, and high levels of contrast agent concentration is recommended $(350-400 \mathrm{mg} / \mathrm{ml})$.

It's estimated that trauma happened in about $8 \%$ of pregnancies, CT is one of the appropriate modalities to diagnose pathologies after trauma such as placental abruption in pregnant patients [48]. This study has some limitations such as the lack of sufficient studies about reducing fetal dose for traumatic patients in the pregnancy period when CT was applied to diagnose pathologies. Also, to the best of my knowledge, no article has been validated Barium Sulphate as an internal shielding in humans.

\section{Conclusion}

Occasionally, diagnostic imaging modalities such as CT during pregnancy are golden standard to diagnose diseases. During CT examination of pregnant patients, we should pay attention to minimize the fetal dose with appropriate approaches, and preserve the diagnostic image quality. In such circumstances, the radiology technologist should perform appropriate CT protocol and shielding during CT examination of a pregnant patient. Furthermore, fetal dose should be estimated by radiation safety officer or medical physicist, and both the physician and the patient must be advised about fetal dose.

\section{Acknowledgment}

Copyright permission was obtained from the journals for using images in the recent study. The author gratefully acknowledges all journals which permitted him to use images in papers that have published in their journals.

\section{References}

1. Chen MM, Coakley FV, Kaimal A, Laros RK. Guidelines for computed tomography and magnetic resonance imaging use during pregnancy and lactation. Obstetrics \& Gynecology. 2008;112(2):333340.
2. Council NR. Health risks from exposure to low levels of ionizing radiation: BEIR VII phase 2. 2006.

3. Wagner LK, Huda W. When a pregnant woman with suspected appendicitis is referred for a CT scan, what should a radiologist do to minimize potential radiation risks? Pediatric radiology. 2004;34(7):589-590.

4. McCollough CH, Schueler BA, Atwell TD, Braun NN, Regner DM, Brown DL, et al. Radiation exposure and pregnancy: when should we be concerned? Radiographics. 2007;27(4):909-917.

5. McCollough CH, Primak AN, Braun N, Kofler J, Yu L, Christner J. Strategies for reducing radiation dose in CT. Radiologic Clinics. 2009;47(1):27-40.

6. Angel E, Wellnitz CV, Goodsitt MM, Yaghmai N, DeMarco JJ, Cagnon $\mathrm{CH}$, et al. Radiation dose to the fetus for pregnant patients undergoing multidetector CT imaging: Monte Carlo simulations estimating fetal dose for a range of gestational age and patient size. Radiology. 2008;249(1):220-227.

7. Reston, Va. practice guideline for imaging pregnant or potentially pregnant adolescents and women with ionizing radiation. American College of Radiology, 2008.

8. Tocilla B. Imaging Pregnant Patients in Different Acute Medical Non-Traumatic Emergencies. A Literature Review. Albanian Journal of Trauma and Emergency Surgery. 2020;4(1):589-602.

9. Leung AN, Bull TM, Jaeschke R, Lockwood CJ, Boiselle PM, Hurwitz LM, et al. An official American Thoracic Society/Society of Thoracic Radiology clinical practice guideline: evaluation of suspected pulmonary embolism in pregnancy. American journal of respiratory and critical care medicine. 2011;184(10):1200-1208.

10. Wieseler KM, Bhargava P, Kanal KM, Vaidya S, Stewart BK, Dighe MK. Imaging in pregnant patients: examination appropriateness. Radiographics. 2010;30(5):1215-1229.

11. Kalra MK, Maher MM, Toth TL, Hamberg LM, Blake MA, Shepard J-A, et al. Strategies for CT radiation dose optimization. Radiology. 2004;230(3):619-628. 
12. Gu J, Xu XG, Caracappa PF, Liu B. Fetal doses to pregnant patients from CT with tube current modulation calculated using Monte Carlo simulations and realistic phantoms. Radiation protection dosimetry. 2013;155(1):64-72.

13. Kennedy E, Iball G, Brettle D. Investigation into the effects of lead shielding for fetal dose reduction in CT pulmonary angiography. The British journal of radiology. 2007;80(956):631-638.

14. Iball GR, Brettle DS. Use of lead shielding on pregnant patients undergoing CT scans: results of an international survey. Radiography. 2011;17(2):102-108.

15. Ryckx N, Sans-Merce M, Schmidt S, Poletti P-A, Verdun FR. The use of out-of-plane high $\mathrm{Z}$ patient shielding for fetal dose reduction in computed tomography: literature review and comparison with Monte-Carlo calculations of an alternative optimisation technique. Physica Medica. 2018;48:156161.

16. Santos FS, Gomez AML, da Silva CAM, do Carmo Santana P, Mourao AP. Analysis of thyroid absorbed dose in cervical CT scan with the use of bismuth shielding. Brazilian Journal of Radiation Sciences. 2019;7(2A). doi.org/10.15392/bjrs. v7i2A.614

17. Ghaznavi H, Momeni Z, Ghaderi S. Assessment of Thyroid Cancer Risk After Cervical Computed Tomography: The Impact of Bismuth Shielding. Disease and Diagnosis. 2021.28;10(2):70-74.

18. Hopper KD, editor Orbital, thyroid, and breast superficial radiation shielding for patients undergoing diagnostic CT. Seminars in Ultrasound, CT and MRI; 2002;23(5):423-427.

19. Hohl C, Wildberger J, Süß C, Thomas C, Mühlenbruch G, Schmidt T, et al. Radiation dose reduction to breast and thyroid during MDCT: effectiveness of an in-plane bismuth shield. Acta Radiologica. 2006;47(6):562-567.

20. Chatterson LC, Leswick DA, Fladeland DA, Hunt MM, Webster ST. Lead versus bismuth-antimony shield for fetal dose reduction at different gestational ages at CT pulmonary angiography. Radiology. 2011;260(2):560-567.
21. Iball G, Kennedy E, Brettle D. Modelling the effect of lead and other materials for shielding of the fetus in CT pulmonary angiography. The British journal of radiology. 2008;81(966):499-503.

22. Yousefzadeh DK, Ward MB, Reft C. Internal barium shielding to minimize fetal irradiation in spiral chest CT: a phantom simulation experiment. Radiology. 2006;239(3):751-758.

23. Kalra MK, Maher MM, Sahani DV, Blake MA, Hahn PF, Avinash GB, et al. Low-dose CT of the abdomen: evaluation of image improvement with use of noise reduction filters-pilot study. Radiology. 2003;228(1):251-256.

24. Silva AC, Lawder HJ, Hara A, Kujak J, Pavlicek W. Innovations in CT dose reduction strategy: application of the adaptive statistical iterative reconstruction algorithm. American Journal of Roentgenology. 2010;194(1):191-199.

25. Sulieman A, Adam H, Elnour A, Tamam N, Alhaili A, Alkhorayef $M$, et al. Patient radiation dose reduction using a commercial iterative reconstruction technique package. Radiation Physics and Chemistry. 2021;178: doi.org/10.1016/j. radphyschem.2020.108996.

26. Greffier J, Hamard A, Pereira F, Barrau C, Pasquier $\mathrm{H}$, Beregi JP, et al. Image quality and dose reduction opportunity of deep learning image reconstruction algorithm for CT: a phantom study. European radiology. 2020;30(7):3951-3959.

27. McLeavy C, Chunara M, Gravell R, Rauf A, Cushnie A, Talbot CS, et al. The future of CT: deep learning reconstruction. Clinical radiology. 2021. doi. org/10.1016/j.crad.2021.01.010

28. Semins MJ, Matlaga BR. Kidney stones during pregnancy. Nature Reviews Urology. 2014;11(3):163-168.

29. Kirubarajan A, Taheri C, Yau M, Aggarwal R, Lam AC, Golda N, et al. Incidence of kidney stones in pregnancy and associations with adverse obstetrical outcomes: a systematic review and meta-analysis of 4.7 million pregnancies. The Journal of Maternal-Fetal \& Neonatal Medicine. 2021: https://doi.org/10.1080/14767058.2021.1878141 
30. Ozbayrak M, Cavdar I, Seven M, Uslu L, Yeyin N, Tanyildizi $\mathrm{H}$, et al. Determining and managing fetal radiation dose from diagnostic radiology procedures in Turkey. Korean journal of radiology. 2015;16(6):1276-1282.

31. Hurwitz LM, Yoshizumi T, Reiman RE, Goodman PC, Paulson EK, Frush DP, et al. Radiation dose to the fetus from body MDCT during early gestation. American Journal of Roentgenology. 2006;186(3):871-876.

32. White WM, Zite NB, Gash J, Waters WB, Thompson W, Klein FA. Low-dose computed tomography for the evaluation of flank pain in the pregnant population. Journal of endourology. 2007;21(11):12551260 .

33. Rodger F, Roditi G, Aboumarzouk OM. Diagnostic accuracy of low and ultra-low dose CT for identification of urinary tract stones: a systematic review. Urologia internationalis. 2018;100(4):375-85.

34. Valovska M-TI, Pais Jr VM. Contemporary best practice urolithiasis in pregnancy. Therapeutic advances in urology. 2018;10(4):127-138.

35. Rob S, Bryant T, Wilson I, Somani B. Ultra-low-dose, low-dose, and standard-dose CT of the kidney, ureters, and bladder: is there a difference? Results from a systematic review of the literature. Clinical radiology. 2017;72(1):11-15.

36. Mancini JG, Ferrandino MN. The impact of new methods of imaging on radiation dosage delivered to patients. Current opinion in urology. 2010;20(2):163-168.

37. Taguchi K, Cho SY, Ng AC, Usawachintachit M, Tan YK, Deng YL, et al. The Urological Association of Asia clinical guideline for urinary stone disease. International Journal of Urology. 2019;26(7):688709 .

38. Tracey M, Fletcher HS, Hollenbeck JI, Sardi A. Appendicitis in pregnancy/discussion. The American Surgeon. 2000;66(6):559-60.

39. Khandelwal A, Fasih N, Kielar A. Imaging of acute abdomen in pregnancy. Radiologic Clinics. 2013;51(6):1005-1022.

40. Wallace CA, Petrov MS, Soybel DI, Ferzoco SJ,
Ashley SW, Tavakkolizadeh A. Influence of imaging on the negative appendectomy rate in pregnancy. Journal of Gastrointestinal Surgery. 2008;12(1):4650.

41. Solomou G, Papadakis A, Damilakis J. Abdominal CT during pregnancy: a phantom study on the effect of patient centring on conceptus radiation dose and image quality. European radiology. 2015;25(4):911-921.

42. Ray J, Chan W. Deep vein thrombosis during pregnancy and the puerperium: a meta-analysis of the period of risk and the leg of presentation. Obstetrical \& gynecological survey. 1999;54(11):169-175.

43. Tan TC, Goh CMY, Tan SSX, Tan LK, Yang Y, Lee LH. Epidemiology of pregnancy-associated pulmonary embolism in South Asian multi-ethnic country: Mortality trends over the last four decades. Journal of Obstetrics and Gynaecology Research. 2021;47(1):174-183.

44. Patel SJ, Reede DL, Katz DS, Subramaniam R, Amorosa JK. Imaging the pregnant patient for nonobstetric conditions: algorithms and radiation dose considerations. Radiographics. 2007;27(6):17051722 .

45. Prakash P, Kalra MK, Kambadakone AK, Pien H, Hsieh J, Blake MA, et al. Reducing abdominal CT radiation dose with adaptive statistical iterative reconstruction technique. Investigative radiology. 2010;45(4):202-210.

46. Laqmani A, Regier M, Veldhoen S, Backhaus A, Wassenberg F, Sehner S, et al. Improved image quality and low radiation dose with hybrid iterative reconstruction with $80 \mathrm{kV}$ CT pulmonary angiography. European journal of radiology. 2014;83(10):1962-1969.

47. Begano D, Söderberg M, Bolejko A. To Use or Not Use Patient Shielding on Pregnant Women Undergoing CT Pulmonary Angiography: A Phantom Study. Radiation Protection Dosimetry. 2020;189(4):458-465.

48. Mendez-Figueroa H, Dahlke JD, Vrees RA, Rouse DJ. Trauma in pregnancy: an updated systematic review. American journal of obstetrics and gynecology. 2013;209(1):1-10. 


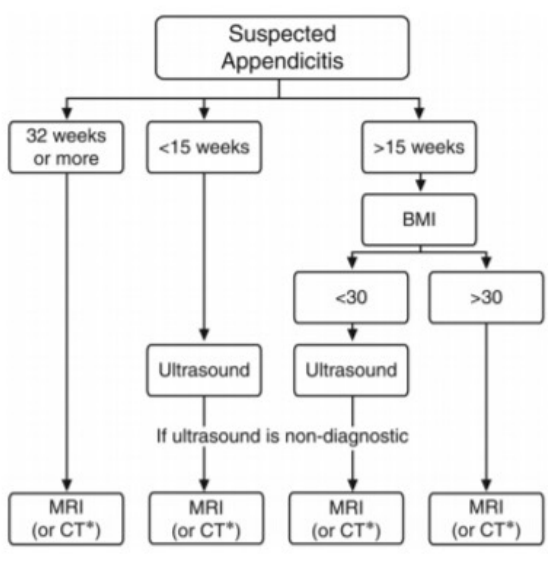

(a)

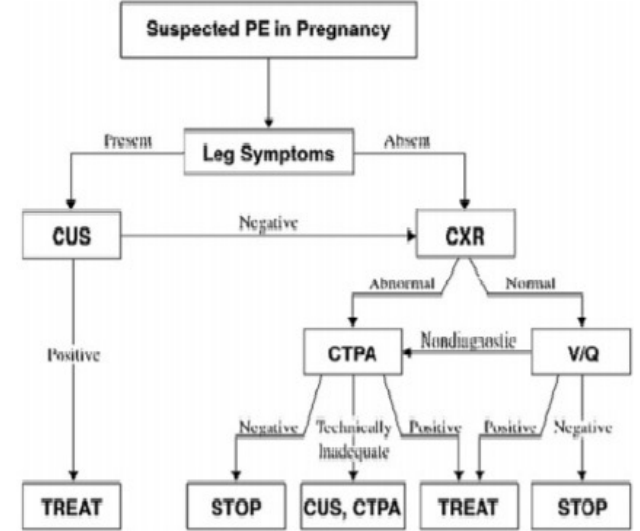

(b)

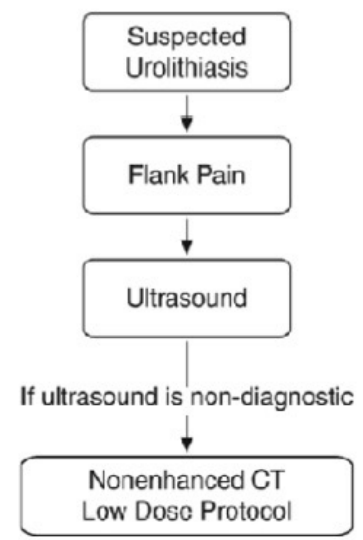

(c)

Figure. 1. Algorithm for performing diagnostic procedures in pregnant patients (BMI: body mass index, ${ }^{*}=$ use $^{*}$ CT if MR imaging is unavailable, CXR: Chest Radiography, CTPA: Computed Tomographic Pulmonary Angiography, DSA: Digital Subtraction Angiography, V/ Q: Ventilation-/Perfusion, CUS: Compression Ultrasound).

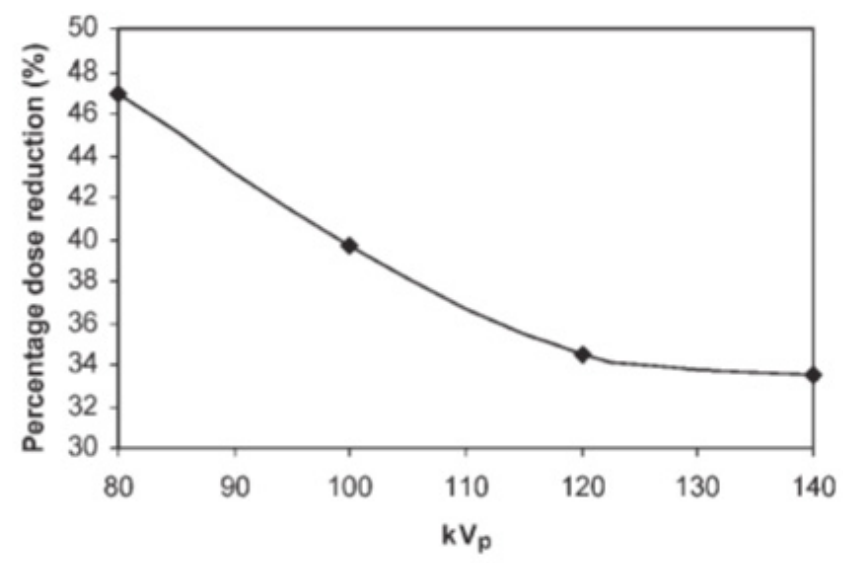

(a)

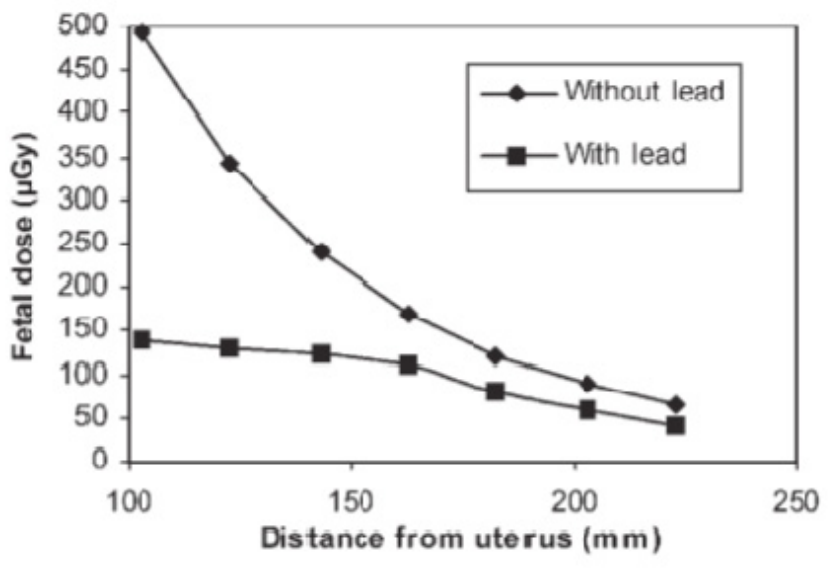

(b)

Figure 2. (a). Percentage of reduction in dose with $0.7 \mathrm{~mm}$ lead for varying $\mathrm{kVp}$, and, (b) The variation in fetal dose based on distance from uterus with and without lead shields. 


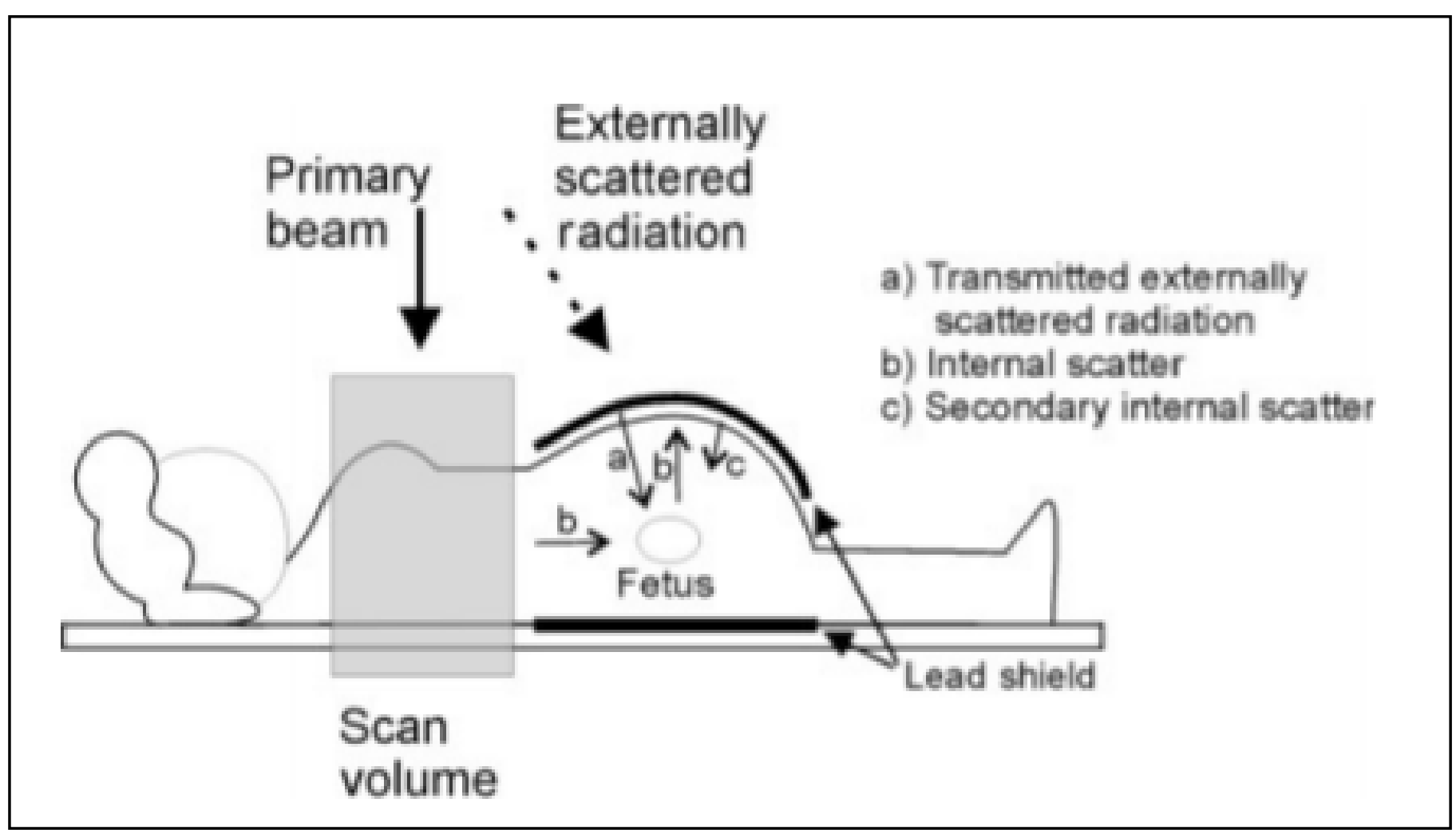

Figure 3. Diagram of sources of scattered radiation that contribute to the fetal dose. 